\title{
COMMUNICATION
}

Cite this: DOI: 10.1039/x0xx00000x

\section{Bacterial meningitis pathogens identified in clinical samples using a SERS DNA detection assay}

\author{
Kirsten Gracie ${ }^{a}$, Diane Lindsay ${ }^{b}$, Duncan Graham $^{a}$, Karen Faulds $^{a} *$ \\ ${ }^{a}$ Centre for Molecular Nanometrology, WestCHEM, Department of Pure and Applied Chemistry, \\ University of Strathclyde, 295 Cathedral Street, Glasgow, G1 1XL
}

Received 00th January 2012, Accepted 00th January 2012

DOI: $10.1039 / \mathrm{x} 0 \mathrm{xx} 00000 \mathrm{x}$

www.rsc.org/

\author{
${ }^{b}$ Scottish Haemophilus Legionella Meningococcus Pneumococcus Reference Laboratory, Scottish \\ Microbiology Reference Laboratory, New Lister Building, 10-16 Alexandra Parade, Glasgow, G31 \\ $2 E R$
}

This communication reports the first demonstration of the detection of Streptococcus pneumoniae and Neisseria meningitidis bacterial DNA extracted from anonymous patient CSF samples and assesses the applicability of a previously developed SERS based DNA detection assay as a platform for the detection of multiple meningitis pathogens from clinical samples.

\section{Introduction}

There is a growing need for DNA detection methods that are both highly selective and sensitive. ${ }^{1}$ The most commonly used method of detection involves fluorescence-based techniques such as the polymerase chain reaction (PCR). ${ }^{2,3}$ An alternative method of choice is surface enhanced Raman spectroscopy (SERS), which is known to be a sensitive, molecularly specific technique with the ability to detect multiple analytes simultaneously due to the characteristic fingerprint spectra obtained. ${ }^{4-6}$ To date, numerous assays have been developed for the direct detection of DNA using SERS. ${ }^{7-12}$ SERS has been shown to successfully detect individual DNA bases; however this does not allow for sequence specific detection as the order of bases is unknown. ${ }^{13}$ To overcome this, dye labels have been incorporated into DNA sequences to aid sequence specific detection. Fluorescent dyes produce characteristic SERS spectra that can be indicative of the presence of target disease DNA. The combination of SERS and fluorescent dye labelled DNA has been used successfully to detect the cystic fibrosis trans-membrane conductance regulator (CFTCR) gene, ${ }^{14}$ sequences coding for methicillin-resistant Staphylococcus aureus (MRSA) ${ }^{12,}{ }^{15}$ and furthermore Vo-Dinh et al. developed a SERS-based assay using "molecular sentinels" for the multiplex detection of two biomarkers for breast cancer. ${ }^{16}$

Meningitis is a result of inflammation of the lining around the spinal cord and brain. The majority of meningitis cases are viral in which case the treatment is directed at limiting the effects of the symptoms. ${ }^{17,}{ }^{18}$ However, bacterial meningitis is more serious in comparison, where urgent medical attention and antibiotic administration is required. The three most common bacterial pathogens associated with meningitis and septicaemia are Neisseria meningitidis, Streptococcus pneumoniae and Haemophilus influenzae. ${ }^{19}$ These are commonly identified using culture-based methods that can be time consuming and at time unreliable. The main issue surrounding the diagnosis of the specific pathogen causing meningitis is that upon admission the patients are immediately administered antibiotics, which can obscure the results obtained from the culture based methods making positive pathogen identification difficult. For successful patient treatment, accurate pathogen identification is required to allow for the correct antibiotics to be administered.

We have previously shown the success of a SERS-based assay for the multiplex detection and quantification of DNA from three pathogens Neisseria meningitidis, Streptococcus pneumoniae and Haemophilus influenzae (Figure 1). ${ }^{\mathbf{2 0}}$ DNA relating to each pathogen was detected individually using the assay with picomolar detection limits. Additionally, PCR was performed on plasmid DNA of each pathogen as proof of concept of the ability of the assay to be used in a clinical setting. This was shown to be successful and, more importantly, with the use of chemometric analysis pathogen quantification was possible using SERS for the first time. Here we report the first study to identify pathogen DNA in patient samples to crucially establish if the assay is suitable for use in hospital laboratories.

\section{Results and Discussion}

Clinical samples from anonymous patients containing two pathogens, Streptococcus pneumoniae and Neisseria meningitidis were provided by the national reference laboratory, SHLMPRL. The mean age of the patients was 43 years with a median of 25 reflecting the very young and old age groups presenting with these disease groups. The samples provided are historical, having been previously analysed by Glasgow Royal Infirmary and have been stored over a long period of time, therefore it must be noted that DNA degradation is possible in some of the samples making pathogen identification using the SERS-based assay difficult. 


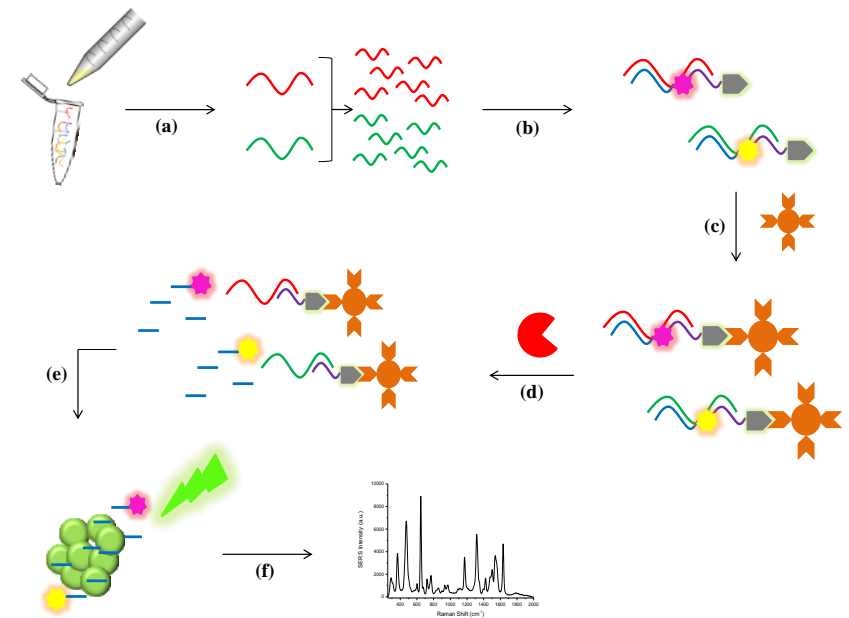

Figure 1 Schematic of the SERS detection assay illustrating each step involved. After the pathogen DNA is extracted from the CSF, PCR is performed (a), which amplifies the amount of each pathogen present to enable further analysis. The amplified pathogen DNA is added to a solution of reporter (blue) and capture (purple) probes for hybridisation (b). As the capture probe possesses a biotin group, streptavidin coated magnetic beads are added to retain the newly formed duplex and remove and excess DNA (c). Lambda exonuclease is added, to digest and release the reporter probe that bears a 5' phosphate to initiate enzymatic DNA digestion, from the duplex allowing the fluorescent label to be free in solution (d). The products of the enzymatic digestion are added to a solution of spermine and silver nanoparticles (e). SERS analysis is then performed and the characteristic SERS spectra of the fluorescent labels are obtained (f).

Using a Qiagen ${ }^{\mathrm{TM}}$ QIAamp DNA mini kit, bacterial DNA was extracted from cerebral spinal fluid (CSF) and blood from patients admitted to hospital showing a variety of symptoms including sepsis and meningitis. Previously used methods for detecting bacterial meningitis pathogens were culture-based techniques that would potentially take up to 36 hours for a positive diagnosis. ${ }^{21,22}$ The time scale for obtaining a positive diagnosis from the clinical samples using the above SERS-based assay (Figure 1) would be in the region of 5 hours.

Prior to the SERS-based assay being performed, PCR was performed on the DNA from clinical samples as previously described. ${ }^{23}$ Positive DNA samples were then used in SERS. Synthetic primers, specific to the pathogens, were used along with the commercially available Qiagen PCR kit to obtain amplified pathogen DNA. Two PCR reactions were performed simultaneously; one contained the pathogen DNA to be amplified and one omitting this from the sample mixture (no-template control, NTC). Following this, the amplified DNA, or the NTC product, was added to the sample mixture containing two probes; capture and reporter probe. A sandwich hybridisation step was performed where both probes hybridise to the single strand of amplified pathogen DNA, if it is present. The capture probe possesses a biotin modification, which when in the presence of streptavidin coated magnetic beads allows for wash steps to be carried out and removal of any excess/nonspecific components from the reaction mixture and retaining only the newly formed duplex. Enzymatic digestion then takes place using the enzyme lambda exonuclease, which digests the fluorescently labelled reporter probe from the duplex, releasing the fluorescent dye label into solution. Again, using the strong interaction between biotin and streptavidin, the supernatant containing the fluorescent dye label is removed and added to a mixture of silver nanoparticles and spermine allowing for subsequent SERS analysis. Obtaining the SERS spectrum of the fluorescent dye label is indicative of the presence of the bacterial meningitis pathogen and therefore providing a positive diagnosis.
In total, 28 samples were analysed, 19 of these samples gave poor results from PCR amplification due to sample degradation however 9 samples produced results to allow for pathogen identification. The pathogen Neisseria meningitidis was detected when the spectrum of the fluorescent dye fluorescein (FAM) was observed. FAM was the fluorescent label attached to the reporter probe, which was complementary to the Neisseria meningitidis DNA sequence. PCR was performed on the clinical samples provided. Results from three of these samples are shown in Figure 2. When Neisseria meningitidis pathogen DNA is present, the characteristic FAM SERS spectrum is observed (red spectra) in each case showing peaks at 635, 1325 and $1635 \mathrm{~cm}^{-1}$. As mentioned above, a control experiment (NTC) was also carried out to confirm SERS intensity is only observed when the pathogen is present (black spectra). A low intensity SERS spectrum is observed, particularly in Figure 2b. This could occur when the reporter probe has not been efficiently removed from the streptavidin beads and some has remained and will produce signal in the final SERS analysis step. Increasing the number of wash steps prior to enzymatic digestion could reduce this. However, the discrimination between the positive sample and the NTC was large and therefore positive identification is possible.
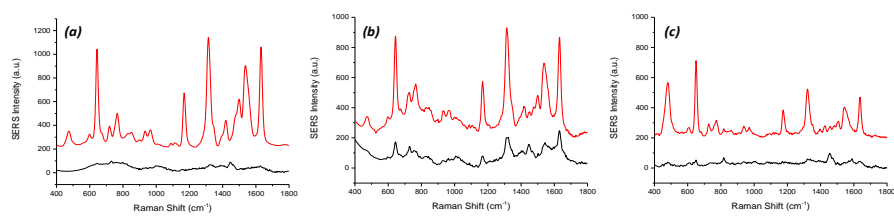

Figure 2 SERS spectra of FAM labelled probe obtained when Neisseria meningitidis pathogen PCR product from DNA extracted from patient samples was present (red) and the no-template control, NTC (black). The three spectra, a-c, are of three different clinical samples analysed using the SERS-based assay. SERS spectra were recorded using an excitation wavelength of $532 \mathrm{~nm}$ with an accumulation of 1 second.

DNA extracted from patient samples containing the second pathogen, Streptococcus pneumoniae, was detected in the same way. To determine the presence of this pathogen, the reporter probe complementary to the Streptococcus pneumoniae DNA sequence was labelled with tetramethylrhodamine (TAMRA) and when the SERS spectrum of TAMRA was observed positive pathogen identification was possible. PCR was again performed prior to the SERS assay, to amplify the amount of Streptococcus pneumoniae pathogen DNA to facilitate SERS detection. Two PCR experiments were performed; one where pathogen DNA was present and another where it was absent (NTC). When the characteristic TAMRA peaks at 1375 and $1650 \mathrm{~cm}^{-1}$ were observed, this was confirmation that the bacterial pathogen, Streptococcus pneumoniae, was present in the clinical sample (red spectra), Figure 3 . As observed with the results from Neisseria meningitidis (Figure 2), peak intensities were observed in the NTC (black spectra), which is a result of the presence of the reporter probe post-washing before SERS analysis. This can be rectified by increasing the number of wash steps in the SERS-based assay. The intensity observed in the TAMRA NTC spectra are higher than those compared to the FAM NTC spectra due to the fact TAMRA has a larger Raman cross section compared to FAM, therefore will produce a higher SERS intensity spectra when present. 

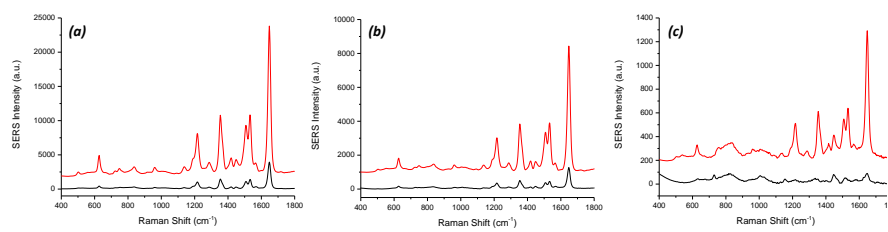

Figure 3 SERS spectra of TAMRA labelled probe obtained when using Streptococcus pneumoniae pathogen PCR product (red) and the no-template control, NTC (black). The three spectra, a-c, are of three different clinical samples analysed using the SERS-based assay. SERS spectra were recorded using an excitation wavelength of $532 \mathrm{~nm}$ with an accumulation of 1 second.

When a patient is admitted presenting symptoms of bacterial meningitis, to provide the most efficient course of treatment it is imperative to provide positive identification of multiple pathogens in a short time scale. The SERS assay has previously been shown to successfully detect three bacterial meningitis pathogens simultaneously, which did not involve clinical samples. The two pathogens; Neisseria meningitidis and Streptococcus pneumoniae present in the clinical samples, were to be detected simultaneously for the first time using the SERS-based assay. The samples provided by the Glasgow Royal Infirmary only contain one pathogen per sample. However, it is important to have the ability to screen for multiple pathogens using multiple probes and fluorescent labels. Therefore, PCR was performed using two approaches: one where the two pathogens were amplified simultaneously in the same reaction mixture and another where amplification of each pathogen occurred in separate reaction tubes and were mixed prior to the assay being performed, results are shown in Figure $4 \mathrm{a}$ and $\mathrm{b}$ respectively. The spectra obtained when the two pathogens were present (green spectra) contain characteristic peaks of both FAM and TAMRA, which are highlighted. Regardless of the chosen method of PCR amplification, both pathogens can be detected simultaneously. However, the simultaneous amplification approach is preferred as it decreases the time taken to obtain a positive result and reduces the amount of samples that are analysed. This is an encouraging result as it demonstrates the assay can be used successfully to detect multiple pathogens in a single patient sample.
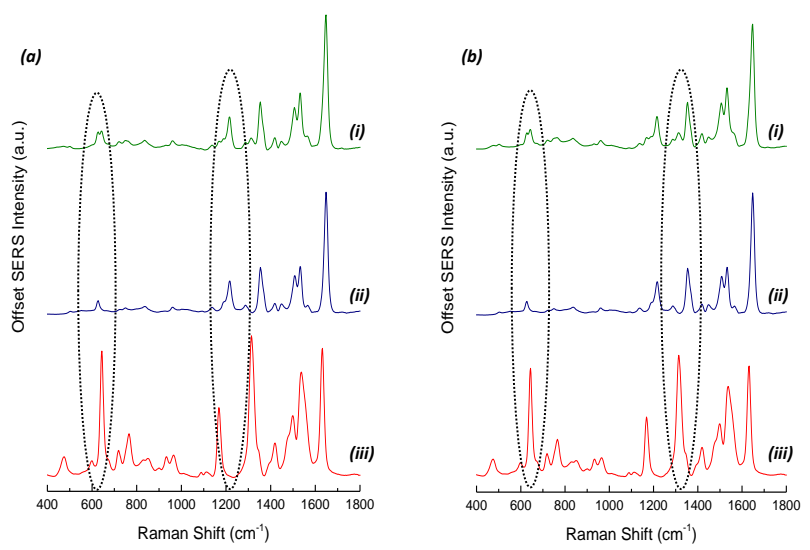

Figure 4 SERS spectra obtained when detecting both Neisseria meningitidis (FAM) and Streptococcus pneumoniae (TAMRA) simultaneously (green spectra). For reference, the characteristic spectra of TAMRA (blue) and FAM (red) are shown. Two PCR methods were used, one where the two pathogens were amplified in the same tube (a) and in separate tubes (b) then mixed prior to the assay being performed. Characteristic peaks of both dyes are highlighted. SERS spectra were recorded using an excitation wavelength of $532 \mathrm{~nm}$ with an accumulation of 1 second.

\section{Conclusions}

This study shows the ability of a SERS-based assay to detect two bacterial meningitis pathogens present in clinical samples of patients with confirmed bacterial meningitis. This was done both individually and simultaneously with equally successful results. This is the first report of bacterial meningitis pathogen DNA extracted from patient clinical samples then amplified by PCR has been detected using SERS. From the 28 clinical samples provided, 9 gave excellent discrimination to allow for pathogen identification using the SERS assay. The assay used is not limited to the detection of bacterial meningitis pathogens since changing the sequences of the capture and reporter probes, a variety of infectious diseases can be detected on a clinical platform.

\section{Notes and references}

Electronic Supplementary Information (ESI) available: [details of any supplementary information available should be included here]. See DOI: $10.1039 / \mathrm{c} 000000 \mathrm{x} /$

1. $\quad$ K. Faulds, R. P. Barbagallo, J. T. Keer, W. E. Smith and D. Graham, Analyst, 2004, 129, 567-568.

2. M. J. Espy, J. R. Uhl, L. M. Sloan, S. P. Buckwalter, M. F. Jones, E. A. Vetter, J. D. C. Yao, N. L. Wengenack, J. E. Rosenblatt, F. R. Cockerill III and T. F. Smith, Clinical Microbiology Reviews, 2006, 19, 165-256.

3. R. T. Ranasinghe and T. Brown, Chemical Communications, 2005, 5487-5502.

4. K. Faulds, F. McKenzie, W. E. Smith and D. Graham, Angewandte Chemie International Edition, 2007, 46, 1829-1831.

5. K. Faulds, R. Jarvis, W. E. Smith, D. Graham and R. Goodacre, Analyst, 2008, 133, 1505-1512.

6. M. Zhong, M. Girolami, K. Faulds and D. Graham, Journal of the Royal Statistical Society: Series C (Applied Statistics), 2011, 60, 187-206.

7. D. Graham, B. J. Mallinder, D. Whitcombe, N. D. Watson and W. E. Smith, Analytical Chemistry, 2002, 74, 1069-1074.

8. R. J. YunWei Charles Cao, and Chad A. Mirkin, Science, 2002, 297, 1536-1540.

9. L. R. Allain and T. Vo-Dinh, Analytica Chimica Acta, 2002, 469 , 149-154.

10. E. Papadopoulou and S. E. J. Bell, Angewandte Chemie International Edition, 2011, 50, 9058-9061.

11. D. van Lierop, K. Faulds and D. Graham, Analytical Chemistry, 2011, 83, 5817-5821.

12. M. M. Harper, J. A. Dougan, N. C. Shand, D. Graham and K. Faulds, Analyst, 2012, 137, 2063-2068.

13. S. E. J. Bell and N. M. S. Sirimuthu, Journal of the American Chemical Society, 2006, 128, 15580-15581.

$14 . \quad$ D. Graham, B. J. Mallinder and W. E. Smith, Angewandte Chemie-International Edition, 2000, 39, 1061-1063.

15. A. MacAskill, D. Crawford, D. Graham and K. Faulds, Analytical Chemistry, 2009, 81, 8134-8140.

16. H. N. Wang and T. Vo-Dinh, Nanotechnology, 2009, 20.

$17 . \quad$ L. Kupila, T. Vuorinen, R. Vainionpaa, V. Hukkanen, R. J. Marttila and P. Kotilainen, Neurology, 2006, 66, 75-80.

$18 . \quad$ R. K. Gupta, J. Best and E. MacMahon, BMJ, 2005, 330, $1132-$ 1135.

19. J. Murray, M. Agócs, F. Serhan, S. Singh, M. Deloria-Knoll, K O'Brien, J. M. Mwenda, R. Mihigo, L. Oliveira, N. Teleb, H. Ahmed, A. Wasley, D. Videbaek, P. Wijesinghe, A. B. Thapa, K. Fox, F. J. Paladin, R. Hajjeh, S. Schwartz, C. V. Beneden, T. Hyde, C. Broome and T. Cherian, MMWR Morb. Mortal. Wkly Rep, 2014, 63, 1159-1162.

$20 . \quad$ K. Gracie, E. Correa, S. Mabbott, J. A. Dougan, D. Graham, R. Goodacre and K. Faulds, Chemical Science, 2014, 5, 1030-1040.

21. X. Wang, M. J. Theodore, R. Mair, E. Trujillo-Lopez, M. du Plessis, N. Wolter, A. L. Baughman, C. Hatcher, J. Vuong, L. 
Lott, A. von Gottberg, C. Sacchi, J. M. McDonald, N. E. Messonnier and L. W. Mayer, Journal of Clinical Microbiology, 2012, 50, 702-708.

22. H. Sadighian and M. R. Pourmand, Iranian Journal of Public Health, 2009, 38, 60-68.

$23 . \quad$ C. E. Corless, M. Guiver, R. Borrow, V. Edward-Jones, A. J. Fox and E. B. Kaczmarski, Journal of Clinical Microbiology 2001, 39, 1553-1558. 